\title{
Induction therapy with paclitaxel and bevacizumab followed by switch maintenance therapy with eribulin in Japanese patients with HER2-negative metastatic breast cancer: a multicenter, collaborative, open-label, phase II clinical study for the SBCCSG 35 investigators
}

Kenichi Inoue $^{1^{*}}$ D, Jun Ninomiya ${ }^{2}$, Tsuyoshi Saito ${ }^{3}$, Kei Kimizuka ${ }^{4}$ and Masafumi Kurosumi ${ }^{5}$

\begin{abstract}
Background: To examine the efficacy and safety of induction therapy with paclitaxel and bevacizumab followed by switch maintenance therapy with eribulin (ISMT) in Japanese patients with HER2-negative metastatic breast cancer $(\mathrm{MBC})$.

Methods: Patients, who had previously undergone a maximum of 2 regimens of chemotherapy, received 3 cycles of induction therapy with paclitaxel $\left(90 \mathrm{mg} / \mathrm{m}^{2}\right.$ intravenously on days 1, 8, and 15 followed by 1-week drug holiday) and bevacizumab (10 mg/kg intravenously after the completion of paclitaxel administration on days 1 and 15). Patients who had complete response, partial response, or stable disease underwent switch maintenance therapy with eribulin $\left(1.4 \mathrm{mg} / \mathrm{m}^{2}\right.$ intravenously on days 1 and 8 followed by 1 -week drug holiday). The primary endpoint was time to treatment failure (TTF) for ISMT.
\end{abstract}

Results: Fifty-one eligible patients (median age: 66 years; range: 35-74) were enrolled: 19 (37.3\%) and 32 (62.7\%) had stage IV and recurrence, respectively, 42 (82.4\%) had visceral metastases, and 45 (88.2\%) received eribulin-38 of whom showed disease progression, and 40 (78.4\%) underwent post therapy. Median TTF was 9.2 months (95\% confidence interval [Cl]: 7.3-11.1), median progression-free survival was 10.7 months (95\% Cl: 9.6-11.8), and median overall survival was 20.0 months (95\% Cl: 16.0-24.0). Relative dose intensity was 97.7\% (range: 33.3-100.0) for induction therapy and was 83.3\% (range: 49.3-100.6\%) for eribulin maintenance therapy. The most common adverse event was alopecia (51 [100\%]) in induction therapy and was peripheral sensory neuropathy (37 [82.2\%]) in eribulin maintenance therapy. Eribulin was effective with manageable tolerability.

Conclusions: ISMT may be a promising therapeutic option for patients with MBC.

Trial registration: UMIN000015971. Registration date: January 1, 2015.

Keywords: Metastatic breast cancer, Eribulin, Paclitaxel, Bevacizumab, Switch maintenance therapy, Metastasis

\footnotetext{
* Correspondence: ino.bad.ken@gmail.com

'Division of Breast Oncology, Saitama Cancer Center, 780 Komuro, Ina-machi,

Kita-adachi-gun, Saitama 362-0806, Japan

Full list of author information is available at the end of the article
}

(c) The Author(s). 2018 Open Access This article is distributed under the terms of the Creative Commons Attribution 4.0 International License (http://creativecommons.org/licenses/by/4.0/), which permits unrestricted use, distribution, and reproduction in any medium, provided you give appropriate credit to the original author(s) and the source, provide a link to the Creative Commons license, and indicate if changes were made. The Creative Commons Public Domain Dedication waiver (http://creativecommons.org/publicdomain/zero/1.0/) applies to the data made available in this article, unless otherwise stated. 


\section{Background}

Approximately $90 \%$ of patients diagnosed with breast cancer undergo surgery for the treatment of their primary malignancy [1], and up to $5 \%$ of patients present with distal metastatic breast cancer $(\mathrm{MBC})$ at the time of diagnosis [2]. Breast cancer recurs in about $40 \%$ of patients who underwent surgery [1], the residual recurrence-free survival rates at 5 and 10 years after adjuvant and neoadjuvant systemic therapy are 89 and $80 \%$, respectively [3], and the median survival of patients with $\mathrm{MBC}$ is 28 months [4]. Therefore, MBC is often incurable despite modern treatments [5]. The goals of treatment are the alleviation of symptoms, extension of survival, and improvement in the quality of life (QOL) of patients [6].

Anthracycline (A)- and taxane (T)-containing regimens (collectively, AT therapy) are preferred chemotherapeutic modalities for patients with $\mathrm{MBC}[7,8]$. Treatments other than AT therapy are frequently selected when breast cancer progressed or recurred after AT therapy was conducted as pre- or postoperative chemotherapy. Nevertheless, chemotherapy after recurrence still remains to be improved.

Eribulin, a nontaxane microtubule dynamics inhibitor that is the first agent in the halichondrion class, has the following unique mechanisms of action among tubulin-targeting compounds: 1) inhibition of microtubule polymerization without depolymerization induction and 2) induction of nonproductive tubulin aggregate formation [9-11]. Eribulin monotherapy has shown efficacy and safety in patients with $\mathrm{MBC}$ who had received anthracyclines and/or taxanes [12-14] and in Japanese counterparts $[15,16]$.

On the other hand, the E2100 Study [17] that is a pivotal study of combined therapy using paclitaxel and bevacizumab (the $\mathrm{PB}$ regimen) in patients with human epidermal growth factor receptor 2 (HER2)-negative MBC demonstrated the efficacy and safety of the regimen as first-line chemotherapy for the study population, and its independent blind review [18] validated them; however, the incidence of grade $3 / 4$ peripheral neuropathy was as high as $23.6 \%$. An open-label phase II study of bevacizumab in combination with weekly paclitaxel in Japanese patients with MBC [19] suggested that the activity and tolerability of the combination therapy as first-line therapy were reproducible in the Japanese patient population; again, the incidence of peripheral neuropathy was high. Our previous cohort study [20] also verified both the high efficacy and safety of the PB regimen but disclosed a high dose reduction rate of $28 \%$ in patients who had received the initial paclitaxel dose of $90 \mathrm{mg} / \mathrm{m}^{2}$. Hence, a need to develop a chemotherapeutic regimen that is less likely to cause dose reductions in paclitaxel emerged. Peripheral neuropathy lowers the QOL of patients with $\mathrm{MBC}$ who are undergoing AT therapy and may cause dose reductions or treatment discontinuation. The reported incidences of peripheral neuropathy have been higher for taxane-based therapy $[17,21,22]$ than for eribulin monotherapy [12-16]. The rational of switching from the former to the latter emerged in expectation of the potential reduction in peripheral neuropathy by eribulin $[16,20]$.

Under the abovementioned clinical circumstances, we conducted induction therapy with paclitaxel and bevacizumab followed by switch maintenance therapy (ISMT) with eribulin alone in expectation that this therapeutic paradigm would more favorably maintain the QOL of patients with MBC than does AT therapy. The present first prospective study on ISMT with eribulin alone intended to examine the efficacy and safety of the paradigm in Japanese patients with MBC.

\section{Methods}

\section{Study design}

The present multicenter, collaborative, open-label, Phase II clinical study of ISMT (SBCCSG 35; University Hospital Medical Information Network identifier: 000015971) was conducted in Japanese patients with MBC. Patients were recruited centrally. All patients provided written informed consent before enrollment. The study protocol was approved by the Institutional or Central Ethics Committee, and the study was conducted in accordance with the Declaration of Helsinki, Good Clinical Practice, and local ethical and legal regulations.

The primary endpoint for ISMT was time to treatment failure (TTF). The secondary endpoints for ISMT were progression-free survival (PFS), the overall response rate (ORR), overall survival (OS), safety, peripheral neuropathy, and the assessment of QOL.

\section{Patients}

At the time of entry, female patients were checked for age, height, body weight, history, complications, HER2/ neu expression, hormone status, hematology, and blood chemistry.

Patients were eligible if they met the following requirements: 1) a female diagnosed with histologically or cytologically confirmed breast cancer; 2) a definite diagnosis of MBC; 3) 20 to 74 years of age; 4) Eastern Cooperative Oncology Group (ECOG) performance status, 0 to 2; 5) measurable lesions according to the response evaluation criteria in solid tumors (RECIST) [23]; 6) major organs of well-conserved functions (bone marrow, liver, kidney, and lungs), i.e., neutrophil count: $\geq 1500 / \mu \mathrm{L}$, platelet count: $80,000 / \mu \mathrm{L}$, hemoglobin: $\geq 9.0 \mathrm{~g} / \mathrm{dL}$, aspartate aminotransferase (AST), alanine aminotransferase (ALT), total bilirubin, and serum creatinine: $\leq 2.5$-fold the upper limit at the site; 7) grade $\leq 1$ peripheral neuropathy at screening; 8) HER2-negative breast cancer; 9) 
an expected survival period of $\geq 3$ months since the day of administration onset; 10) no clinical concerns about electrocardiograms; 11) no history of treatment with paclitaxel, bevacizumab, or eribulin after metastasis; and 12) written informed consent provided by the patient herself. The key exclusion criteria were as follows: systemic infection involving fever $\left(\geq 38.0^{\circ} \mathrm{C}\right)$, history of hypersensitivity to investigational drugs and their solvents, metastasis to the brain requiring treatment, interstitial pneumonia, pulmonary fibrosis, poorly controlled hypertension or diabetes mellitus, active double cancer, history of mental impairment, central nervous system impairment, or cerebrovascular neuropathy, pregnancy, breast-feeding, or women of childbearing age, and patients whom the investigator or subinvestigator considered ineligible.

Patients, who achieved disease control, underwent ISMT until disease progression, development of intolerable toxicities, consent withdrawal, or investigator's discretion to discontinue study treatment. Patients, who showed progressive disease (PD), underwent post therapy.

\section{Treatment}

Patients received 3 cycles of induction therapy with paclitaxel $\left(90 \mathrm{mg} / \mathrm{m}^{2}\right.$ intravenously, once daily, on days 1,8 , and 15 followed by 1-week drug holiday) and bevacizu$\mathrm{mab}(10 \mathrm{mg} / \mathrm{kg}$ intravenously after the completion of paclitaxel administration on days 1 and 15) as PB therapy, followed by 1-week drug holiday on day 22. In patients whose status was rated to be stable disease (SD) or better during cycle 3 of the PB regimen, switch maintenance therapy with eribulin $1.4 \mathrm{mg} / \mathrm{m}^{2}$ alone was conducted for 2 consecutive weeks during which they underwent infusion, once weekly, on days 1 and 8 , as well as 1-week drug holiday at week 3 . This cycle was repeated to the extent possible.

The following anticancer therapies were prohibited during the study period because of their potential effects on the assessment of the present clinical study: hormone therapy, immunotherapy, chemotherapy, radiotherapy, surgery, and systemic steroid therapy.

\section{Assessments \\ Efficacy}

Tumor lesions were monitored at screening, within 10 weeks ( \pm 3 weeks) after the date of first administration, and every 6 weeks ( \pm 3 weeks) after the date of previous monitoring of the lesions thereafter. The date of monitoring could be rescheduled as needed when a deterioration in disease status was suspected. Tumor response was assessed according to Response Evaluation Criteria in Solid Tumors version 1.1 [23]. Best overall response was determined based on the following criteria: complete response (CR), partial response (PR), $\mathrm{PD}, \mathrm{SD}$, and not evaluable (NE). TTF was defined as the time from enrollment to treatment discontinuation in ISMT due to any causes including disease deterioration, treatment-related adverse events (AEs), and death. PFS was defined as the time from enrollment to an event (death or deterioration, whichever earlier) in ISMT. Overall survival (OS) was defined as the time from enrollment to any confirmed all-cause death in ISMT.

\section{Safety}

AEs were graded according to the Common Terminology Criteria for Adverse Events, Japanese version 4.0 [24]. AEs of the worst grade were recorded. Peripheral neuropathy was assessed for its time of development and severity, and the QOL of patients in ISMT was evaluated according to the Functional Assessment of Cancer Therapy/Gynecologic Oncology Group-Neurotoxicity (FACT/GOG-Ntx) questionnaire [25].

\section{Statistical analyses}

The present study was subjected to the per-protocol analysis. Continuous variables are expressed as mean \pm SD or counts and percentage, while categorical variables as median and interquartile range. The Kaplan-Meier estimates (median with 95\% CI) were calculated for TTF, PFS, and OS. Descriptive statistics were calculated to assess safety data. Patients were analyzed on an intent-to-treat basis, and those who received at least one investigational drug were analyzed for safety.

In our prior clinical study [20], median TTF for the PB regimen was 6.2 months (95\% CI: 4.2-8.3). Therefore, we established an expected TTF of 9.0 months and a threshold TTF of 6.2 months in patients with $\mathrm{MBC}$ whose response to treatment is SD or better after 3 cycles (2.8 months) of the $\mathrm{PB}$ regimen. During the entry and follow-up periods of 24 months and 12 months, respectively, 51 patients will endow the study with $80 \%$ power at an $\alpha$ level of 0.05 . The target number of patients was set to 53 in consideration of patients who might be excluded from analysis due to ineligibility. A two-tailed $p$-value of $<0.05$ was considered statistically significant. Wilcoxon's signed rank test was conducted to test differences between the pre- and post-treatment scores of the FACT/GOG-Ntx questionnaire. All statistical analyses were made using SPSS version 19 (IBM, Armonk, NY).

\section{Results}

\section{Patient population}

Between January 23, 2015, and February 25, 2016, a total of 53 Japanese female patients with $\mathrm{MBC}$ were recruited to the present clinical study at 4 medical institutions in Japan. Two of these patients were ineligible (one patient underwent 4 regimens of chemotherapy and another had systemic infection involving fever of $38^{\circ} \mathrm{C}$ ). 
Table 1 Demographic and clinical characteristics of patients at baseline $(N=51)$

\begin{tabular}{l} 
Characteristic \\
\hline Age, years \\
Median \\
Range \\
ECOG performance status \\
0 \\
1 \\
2 \\
Tumor status \\
Stage IV \\
Recurrence \\
Status of menopause \\
Premenopause \\
Postmenopause \\
Histopathological types \\
Common \\
Special \\
Hormone receptor status \\
ER-positive \\
ER-negative \\
PgR-positive \\
PgR-negative \\
Triple-negative (ER, PgR, HER2)
\end{tabular}

Pre- or postoperative treatment

Anthracycline
Paclitaxel
Docetaxel

Hormone therapy

Hormone therapy after recurrence

First-line chemotherapy

Second-line chemotherapy

Anthracycline

Oral 5-fluorouracil

Number of metastases

1

2

3

4

5

6-8

Dominant site of metastases

Visceral

Nonvisceral

Site of metastasis
7 (13.7)

10 (19.6)

$13(25.5)$

$5(9.8)$

$10(19.6)$

$6(11.8)$

$42(82.4)$

9 (17.6)

Table 1 Demographic and clinical characteristics of patients at baseline $(N=51)$ (Continued)

\begin{tabular}{ll}
\hline Characteristic & $\mathrm{n}(\%)$ \\
\hline Bone & $35(68.6)$ \\
Liver & $32(62.7)$ \\
Local lymph node & $27(52.9)$ \\
Lung & $21(41.2)$ \\
Local lesion & $17(33.3)$ \\
Distal lymph node & $15(29.4)$ \\
Pleural effusion & $10(19.6)$ \\
Pleura & $6(11.8)$ \\
Brain & $2(3.9)$ \\
Contralateral mamma & $2(3.9)$ \\
Cardiac effusion & $1(2.0)$ \\
Spleen & $1(2.0)$ \\
Pulmonary lymphangitis & $1(2.0)$ \\
Skin & $1(2.0)$ \\
Adrenal & $1(2.0)$ \\
\hline ECOG Eastern Cooperative Oncology Group, ER estrogen receptor, PgR \\
progesterone receptor, HER2 human epidermal growth factor receptor 2
\end{tabular}

42 (82.4) Therefore, 51 were enrolled in the study; 19 (37.3\%) and 32 9 (17.6) (62.7\%) had stage IV and recurrence, respectively. Patient 38 (74.5) characteristics at baseline are shown in Table 1. The num13 (25.5) ber of patients who underwent first-line chemotherapy was 34; and the number of patients who underwent second-line chemotherapy was 17, of whom 6 and 11 received anthracycline and oral 5-fluorouracil, respectively. None of them 16 (31.4) received both classes of anticancer drugs (Table 1). Among 4 (7.8) them, 43 (84.3\%) were postmenopausal, 32 (62.7\%) had 12 (23.5) MBC, 42 (82.4\%) had visceral metastases, $16(31.4 \%)$ had 5 27 (52.9) or more metastases, 27 (53.0\%) underwent hormone ther36 (70.6) apy previously, as well ass 42 (82.4\%), 38 (74.5\%), and 9 (17.6\%) had estrogen receptor (ER)-positive, progesterone receptor (PgR)-positive, and triple-negative breast cancer, respectively. The most predominant site of metastases was the liver, followed by the lung and local lesion. Fifty-one and 45 patients were assessable for the efficacy and safety of the $\mathrm{PB}$ regimen and eribulin maintenance therapy, respectively.

\section{Study drug exposure}

Fifty-one and 45 patients in the PB regimen and eribulin maintenance therapy received the investigational drugs, respectively; the median of cycles delivered in the latter was 10 cycles (range: 1-29 cycles). The relative dose intensity (RDI) in the former was 97.7\% (range: 33.3-100.0), with 3 dose reductions, 17 dose delays, and 9 dose discontinuations. In contrast, the RDI in the latter was $83.3 \%$ (49.3-100.6\%), with 16 dose reductions, 35 dose delays, 38 dose discontinuations, and 2 combinations with pegylated granulocyte colony-stimulating factor. 


\section{Efficacy of induction therapy followed by switch maintenance therapy}

Among 51 enrolled patients, 45 (88.2\%) underwent maintenance treatment with eribulin alone-38 of whom had disease progression during a median follow-up of 17 months and 7 did not. The following best response rates were obtained (Table 2), and a flow diagram showing best overall responses to ISMT is shown (Fig. 1). In the PB regimen, the ORR was $51.0 \%$ (95\% CI: 36.6-65.3) and the disease control rate (DCR: $\mathrm{CR}+\mathrm{PR}+\mathrm{SD})$ was $90.2 \%$ (95\% CI: 78.6-96.7). In eribulin maintenance therapy, the ORR was $64.4 \%$ (95\% CI: 48.8-78.1) and the DCR was 93.3\% (95\% CI: 81.798.6). Furthermore, the following best overall responses were obtained with respect to 17 patients who underwent second-line chemotherapy: for 6 patients who received anthracycline, 3 each were rated to PR and SD; and 11 patients who received oral 5-fluorouracil, PR, $\mathrm{SD}$, and $\mathrm{PD}$ were 8,2 , and 1 , respectively. The Kaplan-Meier curves for TTF, PFS, and OS in ISMT are shown in Fig. 2. Median TTF was 9.2 months (95\% CI: 7.3-11.1) (Fig. 2a), median PFS was 10.7 months (95\% CI: 9.6-11.8) (Fig. 2b), and median OS was 20.0 months (95\% CI: 16.0-24.0) (Fig. 2c). Waterfall plots (Fig. 3a-c) indicate percent changes in metastatic tumor size (total sum of the longest single dimension for measurable

Table 2 Best overall responses

\begin{tabular}{|c|c|c|}
\hline & Number & Percent \\
\hline \multicolumn{3}{|l|}{ PB regimen $(n=51)$} \\
\hline$C R$ & 0 & 0.0 \\
\hline$P R$ & 26 & 51.0 \\
\hline SD & 20 & 39.2 \\
\hline PD & 5 & 9.8 \\
\hline NE & 0 & 0.0 \\
\hline ORR $(C R+P R)$ & 26 & 51.0 \\
\hline $95 \% \mathrm{Cl}$ & $36.6-65.3$ & \\
\hline $\mathrm{DCR}(\mathrm{CR}+\mathrm{PR}+\mathrm{SD})$ & 46 & 90.2 \\
\hline $95 \% \mathrm{Cl}$ & $78.6-96.7$ & \\
\hline \multicolumn{3}{|c|}{ Maintenance treatment with eribulin $(n=45)$} \\
\hline$C R$ & 0 & 0.0 \\
\hline PR & 29 & 64.4 \\
\hline SD & 13 & 28.9 \\
\hline PD & 3 & 6.7 \\
\hline ORR $(C R+P R)$ & 29 & 64.4 \\
\hline $95 \% \mathrm{Cl}$ & $48.8-78.1$ & \\
\hline $\mathrm{DCR}(\mathrm{CR}+\mathrm{PR}+\mathrm{SD})$ & 42 & 93.3 \\
\hline $95 \% \mathrm{Cl}$ & 81.7-98.6 & \\
\hline
\end{tabular}

$B P$ bevacizumab + paclitaxel, $C R$ complete response, $P R$ partial response, $S D$ stable disease, $P D$ progressive disease, $N E$ not evaluable, $O R R$ overall response rate, $D C R$ disease control rate, $C l$ confidence interval

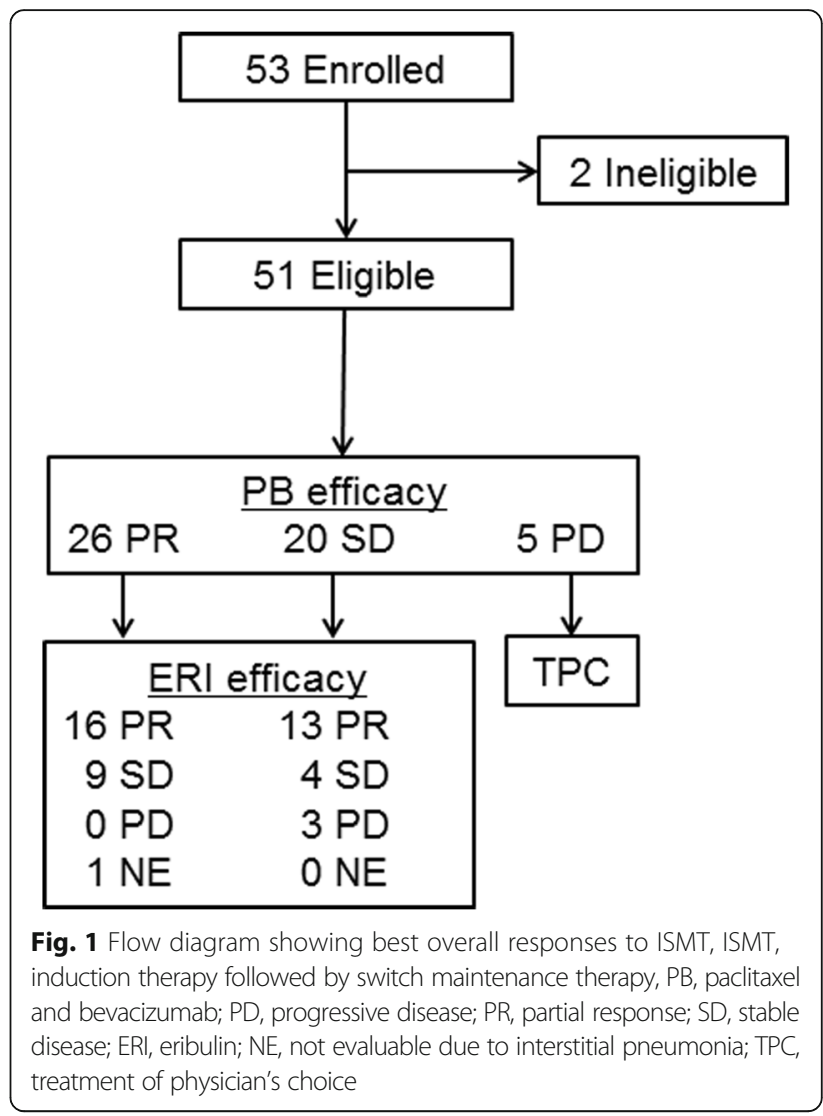

target lesions) from baseline to the maximal tumor shrinkage. In the vast majority of patients with $\mathrm{MBC}$ whose best tumor response was rated to be SD or better, consequently, tumor shrinkage was greater for eribulin maintenance therapy than for the $\mathrm{PB}$ regimen with respect to the following principal organs that showed the elevated incidences of visceral metastases: overall (Fig. 3a), liver (Fig. 3b), and lung (Fig. 3c). The shrinkage rates in the overall, liver, lung, and soft tissue were $93.3 \%$ (42/45), 96.4\% (27/28), and 100.0\% (18/18), respectively.

Among 38 patients who had disease progression, 10 had new lesions ( 6 in the central nervous system and 1 each in the liver, bone, peritoneum, and distal lymph nodes) and 28 had the deterioration of existing lesions.

Forty patients $(78.4 \%)$, who showed disease progression in ISMT, underwent post therapy; 23 of these patients again underwent the $\mathrm{PB}$ regimen (Table 3 ). The DCR was $75.0 \%(15 / 20)$ for the PB regimen.

Regarding the QOL of patients, the scores of the FACT/GOG-Ntx questionnaire lowered significantly ( $p$ $<0.001$ ) from the baseline score of $39.6 \pm 4.8$ to $31.7 \pm$ 8.9 at the end of the $\mathrm{PB}$ regimen and to $30.6 \pm 9.0$ at week 6 of eribulin maintenance therapy. However, no significant difference was found between the $\mathrm{PB}$ regimen and eribulin maintenance therapy. 

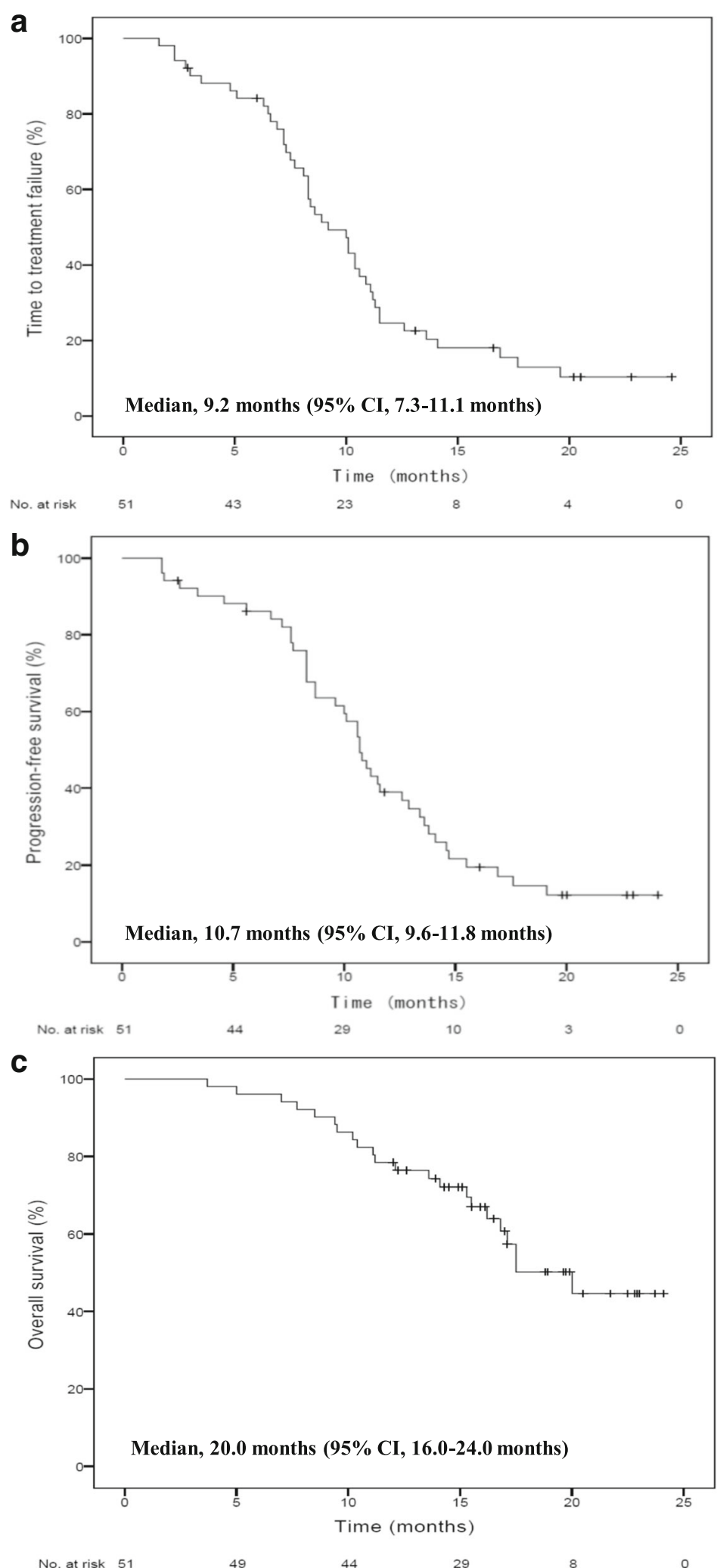

Fig. 2 Kaplan-Meier curves for TTF (a), PFS (b), and OS (c) in ISMT with paclitaxel, bevacizumab, and eribulin, ISMT, induction therapy followed by switch maintenance therapy; TTF, time to treatment failure, PFS, progression-free survival, and OS, overall survival 

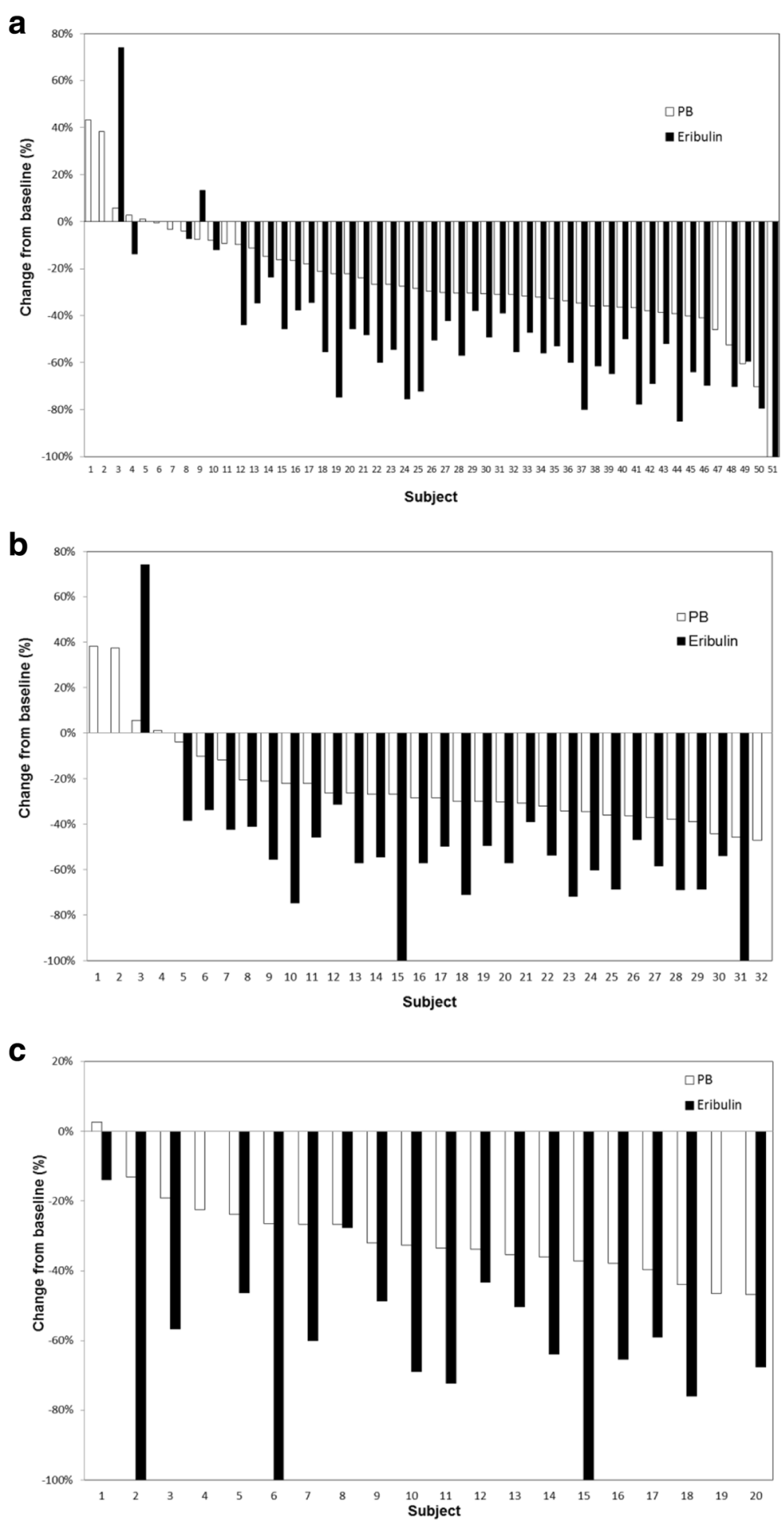

Fig. 3 Waterfall plots of percent changes in metastatic tumor size (total sum of the longest single dimension for measurable target lesions) from baseline to the maximal tumor shrinkage by organ regarding best overall response; (a), overall; (b), liver; (c), lung, PB, paclitaxel and bevacizumab

\section{Safety profile}

All of 51 patients who underwent the $\mathrm{PB}$ regimen and more than $80 \%$ of patients who underwent eribulin maintenance therapy experienced AEs (Table 4). All the
AEs were clinically manageable by dose modifications (dose interruptions/delays or dose reductions) or symptomatic treatment. The most common hematologic AE was anemia in the $\mathrm{PB}$ regimen and eribulin maintenance 
Table 3 Post therapy

\begin{tabular}{lllllllr}
\hline & $\mathrm{n}$ & $\mathrm{PR}$ & L-SD & SD & PD & NE & PD $^{a}$ \\
\hline ADM/EPI + CPA & 3 & & & & 3 & & 1 \\
S1 & 3 & & & 3 & & & 1 \\
PTX + BEV & 23 & 5 & 1 & 9 & 5 & 3 & \\
ERI & 3 & 1 & & & 1 & 1 & 2 \\
DTX & 1 & & & & 1 & & \\
EXE + EVE & 1 & & & & 1 & & \\
CPA & 1 & & & & 1 & & \\
None & 1 & & & & & & \\
Pembrolizumab & 1 & & & & & 1 & \\
Rx to the whole brain & 3 & 1 & & 1 & 1 & &
\end{tabular}

a: Progressive disease in induction therapy

ADM doxorubicin, EPI epirubicin, CPA cyclophosphamide, PTX paclitaxel, BEV bevacizumab, ERI eribulin, DTX docetaxel, EXE exemestane, EVE everolimus, $R x$ radiation, $P R$ partial response, $L-S D$ long-lasting stable disease, $S D$ stable disease, $P D$ progressive disease, $N E$ not evaluable

therapy, being found in 58.8\% (30/51) and 57.8\% (26/45) of patients, respectively. Among nonhematologic AEs, alopecia $(100 \%, 51 / 51)$ and all-grade peripheral sensory neuropathy $(82.2 \%, 37 / 45)$ were most common in patients who underwent the $\mathrm{PB}$ regimen and eribulin maintenance therapy, respectively. The grade of peripheral sensory neuropathy deteriorated and improved in 14 and 3 patients who underwent eribulin maintenance therapy, respectively. Grade 3 peripheral sensory and motor neuropathies in the $\mathrm{PB}$ regimen and eribulin monotherapy were 15.7 and $37.8 \%$, respectively. Grade 4, hematologic or nonhematologic AEs did not develop. No patient died in ISMT.

\section{Discussion}

In clinical practice where oncologists treat patients with $\mathrm{MBC}$ that progressed or recurred after first-line chemotherapy, there is an increasing demand to develop a novel pharmacotherapeutic paradigm that aims at maximizing primary therapy benefit, improving disease control, and conserving an acceptable QOL. The clinical applications of maintenance therapy (i.e., continuation maintenance and switch maintenance) have evolved in such contexts [26]. A diversity of therapeutic armamentariums for maintenance therapy (e.g., chemotherapy, hormone therapy, and targeted therapy) may be tailored to them. Switch maintenance therapy is the introduction of a new and potentially non-cross-resistant agent at the completion of front-line chemotherapy [27] and is intended to maximize the antitumor efficacy of therapy, to minimize treatment-related toxicities, and to maintain the QOL of individual patients. The first pivotal study using this therapeutic strategy, which examined the impact of the timing of therapy, was presented by Fidias et al. for patients with advanced non-small-cell lung cancer [28].

We conducted eribulin maintenance therapy in patients who achieved disease control with the $\mathrm{PB}$ regimen in an attempt to minimize cumulative toxicities and drug resistance associated with the prolonged administration of the same drugs [29] in first-line chemotherapy and to increase the possibility of benefiting from maintenance therapy [30] with eribulin. This therapeutic devisal translated into good median TTF, PFS, and OS, although the study patient population was severely affected by $\mathrm{MBC}$ as evidenced by the elevated proportions of patients (82.4\%) who had visceral metastases and 4 or more metastases $(41.2 \%)$ at baseline.

Concretely, median PFS of 10.7 months, and median OS of 20.0 months in the present study are longer than 2 to 7 months in PFS [12-16] and 9 to 13 months in OS [12-16] that were reported in previous clinical studies of eribulin monotherapy in patients with pretreated $\mathrm{MBC}$ [12-16]. These clinical outcomes from ISMT were favored by the sequential combination of a highly effective chemotherapy (i.e., the PB regimen) and monochemotherapy that is less likely to provoke peripheral neuropathy (i.e., eribulin monotherapy). The present study successfully met the statistical requirements. Namely, median TTF was 9.2 months (95\% confidence interval [CI]: 7.3-11.1), thus successfully exceeded the established threshold time of 6.2 months. Furthermore, eribulin maintenance therapy contributed to the acceptable preservation of the QOL of patients, which was indicated by the absence of statistical significance in the scores of the FACT/GOG-Ntx questionnaire between the $\mathrm{PB}$ regimen and eribulin maintenance therapy.

The waterfall plot analysis of maximal changes in metastatic tumor size by organ revealed the absence of increased or unchanged tumor size in more than $80 \%$ of patients who underwent ISMT according to the protocol despite switching to treatment with eribulin alone.

The present study on ISMT is the first prospective clinical study on switch maintenance therapy with eribulin alone. To date, a number of studies on maintenance therapy after induction therapy with diversified chemotherapeutic agents have been conducted in pretreated patients with MBC. For example, Gligorov et al. [31] conducted a randomized, open-label, phase 3 clinical study (IMELDA) in patients with HER2-negative MBC, in which first-line docetaxel $\left(75-100 \mathrm{mg} / \mathrm{m}^{2}\right)$ and bevacizumab $(15 \mathrm{mg} / \mathrm{kg})$ was followed by maintenance bevacizumab (15 mg/kg) alone or in combination with capecitabine $\left(1000 \mathrm{mg} / \mathrm{m}^{2}\right)$. Consequently, median PFS was 11.9 months for capecitabine plus bevacizumab and was 4.3 months for bevacizumab alone. Furthermore, median OS was 39.0 months for capecitabine plus 
Table 4 Most common adverse events by CTCAE grade (incidence of all grades: $\geq 15 \%$ )

\begin{tabular}{|c|c|c|c|c|}
\hline \multirow[t]{2}{*}{ Adverse events } & \multicolumn{4}{|c|}{ Severity, n (\%) } \\
\hline & All grades & Grade 1 & Grade 2 & Grade 3 \\
\hline \multicolumn{5}{|l|}{ Hematologic } \\
\hline \multicolumn{5}{|l|}{ PB regimen $(n=51)$} \\
\hline Anemia & $30(58.8)$ & $18(35.3)$ & $9(17.6)$ & $3(5.9)$ \\
\hline Leukopenia & $21(41.2)$ & $10(19.6)$ & $8(15.7)$ & $3(5.9)$ \\
\hline Neutropenia & $19(37.3)$ & $2(3.9)$ & $9(17.6)$ & $8(15.7)$ \\
\hline \multicolumn{5}{|l|}{ Eribulin maintenance therapy $(n=45)$} \\
\hline Anemia & $26(57.8)$ & $21(46.7)$ & $4(8.9)$ & $1(2.2)$ \\
\hline Leukopenia & $24(53.3)$ & $9(20.0)$ & $10(22.2)$ & $5(11.1)$ \\
\hline Neutropenia & $23(51.1)$ & $2(4.4)$ & $12(26.7)$ & $9(20.0)$ \\
\hline \multicolumn{5}{|l|}{ Nonhematologic } \\
\hline \multicolumn{5}{|l|}{ PB regimen $(n=51)$} \\
\hline Alopecia & $51(100.0)$ & $2(3.9)$ & $49(96.1)$ & - \\
\hline Peripheral sensory neuropathy & $37(72.5)$ & $28(54.9)$ & $8(15.7)$ & $1(2.0)$ \\
\hline Increased AST & $15(29.4)$ & $13(25.5)$ & $1(2.0)$ & $1(2.0)$ \\
\hline Dysgeusia & $15(29.4)$ & $15(29.4)$ & $0(0.0)$ & - \\
\hline Epistaxis & $15(29.4)$ & $15(29.4)$ & $0(0.0)$ & $0(0.0)$ \\
\hline Fatigue & $12(23.5)$ & $10(19.6)$ & $2(3.9)$ & $0(0.0)$ \\
\hline Arthralgia & $12(23.5)$ & $12(23.5)$ & $0(0.0)$ & $0(0.0)$ \\
\hline Rash & $12(23.5)$ & $12(23.5)$ & $0(0.0)$ & $0(0.0)$ \\
\hline Increased ALT & $10(19.6)$ & $8(15.7)$ & $2(3.9)$ & $0(0.0)$ \\
\hline Anorexia & $10(19.6)$ & $7(13.7)$ & $3(5.9)$ & $0(0.0)$ \\
\hline Nail discoloration & $10(19.6)$ & 10 (19.6) & - & - \\
\hline Peripheral motor neuropathy & $9(17.6)$ & $8(15.7)$ & $0(0.0)$ & $0(0.0)$ \\
\hline Myalgia & $8(15.7)$ & $8(15.7)$ & $0(0.0)$ & $0(0.0)$ \\
\hline \multicolumn{5}{|l|}{ Eribulin maintenance therapy $(n=45)$} \\
\hline Peripheral sensory neuropathy & $37(82.2)$ & $18(40.0)$ & $17(37.8)$ & $2(4.4)$ \\
\hline Increased AST & $25(55.6)$ & $21(46.7)$ & $3(6.7)$ & $1(2.2)$ \\
\hline Dysgeusia & $16(35.6)$ & $13(28.9)$ & $3(6.7)$ & - \\
\hline Increased ALT & $16(35.6)$ & $14(31.1)$ & $2(4.4)$ & $0(0.0)$ \\
\hline Alopecia & $15(33.3)$ & $8(17.8)$ & $7(15.6)$ & - \\
\hline Fatigue & $14(31.1)$ & $10(22.2)$ & $4(8.9)$ & $0(0.0)$ \\
\hline Anorexia & $14(31.1)$ & $11(24.4)$ & $3(6.7)$ & $0(0.0)$ \\
\hline Peripheral motor neuropathy & $13(28.9)$ & $10(22.2)$ & $3(6.7)$ & $0(0.0)$ \\
\hline Increased creatinine & $13(28.9)$ & $12(26.7)$ & $1(2.2)$ & $0(0.0)$ \\
\hline Nausea & $11(24.4)$ & $11(24.4)$ & $0(0.0)$ & $0(0.0)$ \\
\hline Arthralgia & $10(22.2)$ & $9(20.0)$ & $1(2.2)$ & $0(0.0)$ \\
\hline Myalgia & $9(20.0)$ & $7(15.6)$ & $2(4.4)$ & $0(0.0)$ \\
\hline Nail loss & $9(20.0)$ & $4(8.9)$ & $5(11.1)$ & - \\
\hline Constipation & $8(17.8)$ & $5(11.1)$ & $3(6.7)$ & $0(0.0)$ \\
\hline Epistaxis & $7(15.6)$ & $7(15.6)$ & $0(0.0)$ & $0(0.0)$ \\
\hline Nail discoloration & $7(15.6)$ & 7 (15.6) & - & - \\
\hline
\end{tabular}

CTCAE common terminology criteria for adverse events, $P B$ paclitaxel + bevacizumab, AST aspartate transaminase, ALT alanine transaminase; -, no definition 
bevacizumab and was 23.7 months for bevacizumab only. Thus, the addition of capecitabine to bevacizumab maintenance therapy after taxane-based induction treatment for patients with HER2-negative MBC afforded significant and clinically meaningful improvements in both PFS and OS. Furthermore, Alba et al. [32] conducted a multicenter, randomized, phase III control study in patients with $\mathrm{MBC}$ to assess first-line induction chemotherapy with doxorubicin $\left(75 \mathrm{mg} / \mathrm{m}^{2}\right)$ and docetaxel $\left(100 \mathrm{mg} / \mathrm{m}^{2}\right)$ followed by maintenance therapy consisting of pegylated liposomal doxorubicin (PLD; $40 \mathrm{mg} / \mathrm{m}^{2}$ ) or observation. Consequently, median PFS was 8.4 months for PLD against 5.1 months for observation and median OS was 24.8 months for PLD against 22.0 months for observation. In previous clinical studies on maintenance therapy after first-line induction therapy [30-34], median PFSs ranged between 4 and 12 months and median OSs ranged between 23 and 39 months. Hence, the results from ISMT are comparable to these clinical outcomes despite the severer clinical background of our patient population.

The concept of switching from a chemotherapy to another with greater tolerability emerged as described in the IMELDA study in patients with HER2-negative MBC [31]_"Switching to a more tolerable chemotherapy, such as capecitabine, with a different mechanism of action, while continuing VEGF inhibition, might be a more effective treatment strategy." In the present study, we intended to examine the efficacy and safety of ISMT in which eribulin was used as switch maintenance therapy instead of capecitabine in the same patient population and found the following: 1) none of patients rated to $\mathrm{PR}$ in the $\mathrm{PB}$ regimen experienced disease progression; 2) switch maintenance therapy with eribulin alone was well tolerated (i.e., peripheral neuropathy as assessed with the FACT/GOG-Ntx questionnaire did not deteriorate) in consistency with previous clinical studies of eribulin therapy [12-16]; and 3) the incidence of alopecia drastically lowered from 100\% with the PB regimen to $33.3 \%$ with switch maintenance therapy with eribulin alone.

The present study has several limitations. First, sample size is relatively small, which impedes a robust conclusion equivalent to that is drawn from a large-scale clinical study. However, our study was endowed with sufficient statistical power and is the first to provide valid clinical evidence on eribulin maintenance therapy after the PB regimen. Second, our study is not a randomized controlled clinical study and is therefore of lower evidence level; nevertheless, ISMT is a novel chemotherapeutic paradigm that may be beneficial for patients with $\mathrm{MBC}$ who are under critical conditions after tumor progression or recurrence. Third, switch maintenance therapy was conducted with eribulin alone, not in combination with paclitaxel or bevacizumab, in an attempt to precisely assess its pharmacological effects on breast cancer that progressed or recurred after the $\mathrm{PB}$ regimen. Consequently, inter-regimen comparisons were precluded for maintenance therapy.

\section{Conclusion}

We present the first prospective clinical study on induction therapy with paclitaxel and bevacizumab that was followed by switch maintenance therapy with eribulin alone in Japanese patients with HER2-negative metastatic breast cancer. This therapeutic regimen is effective, safe, and feasible, and therefore may be considered as a promising therapeutic option for patients with MBC that progressed or recurred after first-line chemotherapy.

\section{Abbreviations}

AEs: adverse events; ALT: alanine aminotransferase; AST: aspartate aminotransferase; AT therapy: anthracycline and taxane therapy; Cl: confidence interval; CR: complete response; DCR: disease control rate; ECOG: Eastern Cooperative Oncology Group; ER: estrogen receptor; FACT/ GOG-Ntx: Functional Assessment of Cancer Therapy/Gynecologic Oncology Group-Neurotoxicity; HER2: human epidermal growth factor receptor 2;

ISMT: induction therapy followed by switch maintenance therapy;

IV: intravenous; MBC: metastatic breast cancer; NE: not evaluable; ORR: overall response rate; OS: overall survival; PB regimen: paclitaxel and bevacizumab regimen; PD: progressive disease; PFS: progression-free survival; PgR: progesterone receptor; PLD: pegylated liposomal doxorubicin; PR: progressive disease; QOL: quality of life; RDI: relative dose intensity; SBCCSG: Saitama Breast Cancer Clinical Study Group; SD: stable disease; SD: standard deviation; TTF: time to treatment failure; UMIN: University Hospital Medical Information Network

\section{Acknowledgements \\ The authors thank Satoshi Sakima, MD, for his gracious review of the manuscript.}

\section{Funding}

The present study was supported by the National Cancer Center Research and Development Fund (23-A-16, 23-A-17, 26-A-4) and Health and Labour Sciences Research Expenses for Commission, Applied Research for Innovative Treatment of Cancer from the Ministry of Health, Labour and Welfare (H26-applied-general-043,046). Furthermore, this research was supported by the Practical Research for Innovative Cancer Control (15ck0106046h0002, 15ck0106049h0002) from Japan Agency for Medical Research and Development, AMED. The funding source had no role in the design, data collection, interpretation of data, or writing of the manuscript for the present clinical study.

\section{Availability of data and materials}

The raw data generated and analyzed during this study are not publicly available due to appropriate protection of patient personal information but are available from the corresponding author on reasonable request.

\section{Authors' contributions}

$\mathrm{KI}$ conceived the study, participated in the design, and drafted the manuscript. $\mathrm{KI}$ and $\mathrm{MK}$ conducted the data analysis. Kl, JN, TS, and KK played a significant role in the acquisition and interpretation of data and in the critical revision of the manuscript for important intellectual content. All authors read and approved the final manuscript.

\section{Ethics approval and consent to participate}

All patients provided written informed consent before enrollment. The data were used only for research. The study protocol was approved by the following ethics bodies: for Saitama Cancer Institutional Review Board; for Ninomiya Hospital, Central Ethics Committee at Saitama Cancer Center; for 
Japanese Red Cross Saitama Hospital, Ethics Committee; and for Kasukabe Medical Center, Ethics Committee.

\section{Competing interests}

The authors declare that they have no competing interests.

\section{Publisher's Note}

Springer Nature remains neutral with regard to jurisdictional claims in published maps and institutional affiliations.

\section{Author details}

'Division of Breast Oncology, Saitama Cancer Center, 780 Komuro, Ina-machi, Kita-adachi-gun, Saitama 362-0806, Japan. ²Department of Breast Surgery, Ninomiya Hospital, 2-22-23 Shinei, Soka-shi, Saitama 340-0056, Japan. ${ }^{3}$ Department of Breast Surgery, Japanese Red Cross Saitama Hospital, 1-5 Shintoshin, Chuo-ku, Saitama-shi, Saitama 330-8553, Japan. ${ }^{4}$ Department of Breast Surgery, Kasukabe Medical Center, 6-7-1 Chuo, Kasukabe-shi, Saitama, Japan. ${ }^{5}$ Department of Pathology, Saitama Cancer Center, 780 Komuro, Ina-machi, Kita-adachi-gun, Saitama 362-0806, Japan.

\section{Received: 1 November 2017 Accepted: 25 May 2018}

Published online: 20 June 2018

\section{References}

1. Bonadonna G, Hortobagyi GN, Gianni AM. Textbook of breast cancer, a clinical guide to therapy. London: Taylor \& Fancis; 1997.

2. Jemal A, Bray F, Center MM, Ferlay J, Ward E, Forman D. Global cancer statistics. CA Cancer J Clin. 2011:61:69-90.

3. Brewster AM, Hortobagyi GN, Broglio KR, Kau SW, Santa-Maria CA, Arum B, et al. Residual risk of breast cancer recurrence 5 years after adjuvant therapy. J Natl Cancer Inst. 2008;100:1179-83.

4. Yamamoto N, Watanabe T, Katsumata N, Omuro Y, Ando M, Fukuda H, et al. Construction and validation of a practical prognostic index for patients with metastatic breast cancer. J Clin Oncol. 1998:16:2401-8.

5. McGuire A, Brown JA, Kerin MJ. Metastatic breast cancer: the potential of miRNA for diagnosis and treatment monitoring. Cancer Metastasis Rev. 2015;34:145-55.

6. Hortobagyi GN. Treatment of breast cancer. N Engle J Med. 1998;339: 974-84

7. NCCN Clinical Practice Guidlines in Oncology Breast Cancer Version 1.2018 Available at https://www.nccn.org/professionals/physician_gls/pdf/breast. pdf. Accessed 31 May 2018.

8. Cardoso F, Costa A, Senkus E, Aapro M, André F, Barrios CH, et al. 3rd ESOESMO International Consensus Guidelines for Advanced Breast Cancer (ABC 3). Ann Oncol. 2017;28:3111.

9. Towle MJ, Salvato KA, Budrow J, Wels BF, Kuznetsov G, Aalfs KK, et al. In vitro and in vivo anticancer activities of synthetic macrocyclic ketone analogues of halichondrin B. Cancer Res. 2001;61:1013-21.

10. Smith JA, Wilson L, Azarenko O, Zhu X, Lewis BM, Littlefield BA, et al. Eribulin binds at microtubule ends to a single site on tubulin to suppress dynamic instability. Biochemistry. 2010;49:1331-7.

11. Kuznetsov G, Towle MJ, Cheng H, Kawamura T, TenDyke K, Liu D, et al Induction of morphological and biochemical apoptosis following prolonged mitotic blockage by halichondrin B macrocyclic ketone analog E7389. Cancer Res. 2004;64:5760-6.

12. Vahdat LT, Pruitt B, Fabian CJ, Rivera RR, Smith DA, Tan-Chiu E, et al. Phase II study of eribulin mesylate, a halichondrin B analog, in patients with metastatic breast cancer previously treated with an anthracycline and a taxane. J Clin Oncol. 2009;27:2954-61.

13. McIntyre K, O'Shaughnessy J, Schwartzberg L, Glück S, Berrak E, Song JX, et al. Phase II study of eribulin mesylate as first-line therapy for locally recurrent or metastatic human epidermal growth factor receptor 2-negative breast cancer. Breast Cancer Res Treat. 2014;146:321-8.

14. Cortes J, O'Shaughnessy J, Loesch D, Blum JL, Vahdat LT, Petrakova K, et al. Eribulin monotherapy versus treatment of physician's choice in patients with metastatic breast cancer (EMBRACE): a phase 3 open-label randomised study. Lancet. 2011:377:914-23.

15. Aogi K, Iwata H, Masuda N, Mukai H, Yoshida M, Rai Y, et al. A phase II study of eribulin in Japanese patients with heavily pretreated metastatic breast cancer. Ann Oncol. 2012;23:1441-8.
16. Inoue K, Saito T, Okubo K, Kimizuka K, Yamada H, Sakurai T, et al. Phase II clinical study of eribulin monotherapy in Japanese patients with metastatic breast cancer who had well-defined taxane resistance. Breast Cancer Res Treat. 2016;157:295-305.

17. Miller K, Wang M, Gralow J, Dickler M, Cobleigh M, Perez EA, et al. Paclitaxel plus bevacizumab versus paclitaxel alone for metastatic breast cancer. N Engl J Med. 2007;357:2666-76.

18. Gray R, Bhattacharya S, Bowden C, Miller K, Comis RL. Independent review of E2100: a phase III trial of bevacizumab plus paclitaxel versus paclitaxel in women with metastatic breast cancer. J Clin Oncol. 2009;27:4966-72.

19. Aogi K, Masuda N, Ohno S, Oda T, Iwata H, Kashiwaba M, et al. First-line bevacizumab in combination with weekly paclitaxel for metastatic breast cancer: efficacy and safety results from a large, open-label, single-arm Japanese study. Breast Cancer Res Treat. 2011;129:829-38.

20. Yamada H, Inoue K, Nagai SE, Nakai M, Fumio Arisawa F, et al. A real-world retrospective cohort study of combined therapy with bevacizumab and paclitaxel in Japanese patients with metastatic breast. J Nippon Med Sch. 2017:84:215-23.

21. Jones SE, Erban J, Overmoyer B, Budd GT, Hutchins L, Lower E, et al. Randomized phase III study of docetaxel compared with paclitaxel in metastatic breast cancer. J Clin Oncol. 2005;23:5542-51.

22. Gradishar WJ, Tjulandin S, Davidson N, Shaw H, Desai N, Bhar P, et al. Phase III trial of nanoparticle albumin-bound paclitaxel compared with polyethylated castor oil-based paclitaxel in women with breast cancer. J Clin Oncol. 2005;23:7794-803.

23. Eisenhauer EA, Therasse P, Bogaerts J, Schwartz LH, Sargent D, Ford R, et al. New response evaluation criteria in solid tumors: revised RECIST guideline (version 1.1). Eur J Cancer. 2009:45:228-47.

24. National Cancer Institute Common Terminology Criteria for Adverse Events version 4.0-Japan Clinical Oncology Group. Available at http://www.jcog.jp/ doctor/tool/CTCAEv4J 20150910.pdf. Accessed 15 Sep 2015.

25. Calhoun EA, Welshman EE, Chang CH, Lurain JR, Fishman DA, Hunt TL, et al. Psychometric evaluation of the functional assessment of Cancer therapy/ gynecologic oncology group-neurotoxicity (fact/GOG-Ntx) questionnaire for patients receiving systemic chemotherapy. Int J Gynecol Cancer. 2003;13: $741-8$.

26. Blais N, Kassouf E. Maintenance therapies for non-small cell lung cancer. Front Oncol. 2014;4:1-5.

27. Gerber DE, Schiller JH. Maintenance chemotherapy for advanced non-smallcell lung cancer: new life for an old idea. J Clin Oncol. 2013;31:1009-20.

28. Fidias PM, Dakhil SR, Lyss AP, Loesch DM, Waterhouse DM, Bromund JL, et al. Phase III study of immediate compared with delayed docetaxel after front-line therapy with gemcitabine plus carboplatin in advanced nonsmall-cell lung cancer. J Clin Oncol. 2009:27:591-8.

29. Gennari A1, Stockler M, Puntoni M, Sormani M, Nanni O, Amadori D, et al. Duration of chemotherapy for metastatic breast cancer: a systematic review and meta-analysis of randomized clinical trials. J Clin Oncol. 2011;29:2144-9.

30. Park $\mathrm{YH}$, Jung $\mathrm{KH}$, Im SA, Sohn JH, Ro J, Ahn JH, et al. Phase III, multicenter, randomized trial of maintenance chemotherapy versus observation in patients with metastatic breast cancer after achieving disease control with six cycles of gemcitabine plus paclitaxel as first-line chemotherapy: KCSGBR07-02. J Clin Oncol. 2013;31:1732-9.

31. Gligorov J, Doval D, Bines J, Alba, Cortes P, Pierga JY, et al. Maintenance capecitabine and bevacizumab versus bevacizumab alone after initial firstline bevacizumab and docetaxel for patients with HER2-negative metastatic breast cancer (IMELDA): a randomised, open-label, phase 3 trial. Lancet Oncol. 2014;15:1351-60.

32. Alba E, Ruiz-Borrego M, Margelí M, Rodríguez-Lescure A, Sánchez-Rovira P, Ruiz A, et al. Maintenance treatment with pegylated liposomal doxorubicin versus observation following induction chemotherapy for metastatic breast cancer: GEICAM 2001-01 study. Breast Cancer Res Treat. 2010;122:169-76.

33. Bisagni G, Musolino A, Panebianco M, De Matteis A, Nuzzo F, Ardizzoni A, et al. The breast Avastin trial: phase II study of bevacizumab maintenance therapy after induction chemotherapy with docetaxel and capecitabine for the first-line treatment of patients with locally recurrent or metastatic breast cancer. Cancer Chemother Pharmacol. 2013:71:1051-7.

34. Surmeli ZG, Varol U, Cakar B, Degirmenci M, Arslan C, Piskin GD, et al. Capecitabine maintenance therapy following docetaxel/capecitabine combination treatment in patients with metastatic breast cancer. Oncol Lett. 2015:10:2598-602. 